Indian J. Phys., Vol. 85, No. 7, pp. 1215, July, 2011

\title{
Erratum
}

\section{Title: Chemical equilibration of QGP enforcing baryon number conservation}

\author{
Author: A Sen
}

Ref. : Indian J. Phys. Volume 85, No. 6, pp. 781-785, June, 2011

(1) Correct e-mail address of the Author is abhijisen@yahoo.co.in

(2) The printer committed a serious mistake by printing a part of Figure 1. The Corrected Figure 1 with caption should be as follows :

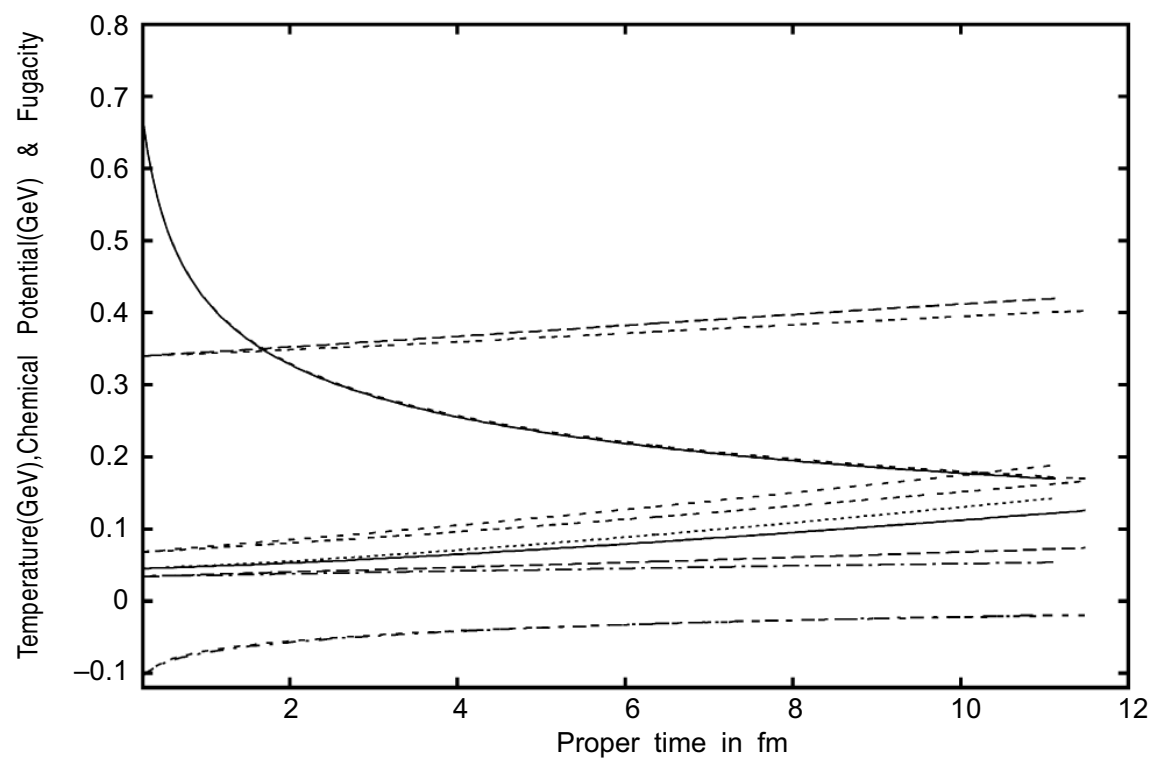

Figure 1. The temperature, chemical potential and non-equilibrium fugacity variations for RHIC initial conditions. Sequentially from top we have A] For decaying curves variations of : 1. Temperature (-QFCP), Temperature $(+Q F C P) \& B$ ] for rising curves we have gluon fugacity (+ QFCP), gluon fugacity (- QFCP), light quark fugacity $(+Q F C P)$, curve $3 b$ : light quark fugacity (- QFCP), light antiquark fugacity (+ QFCP), light antiquark fugacity (- QFCP), strange quark fugacity (- QFCP), strange quark fugacity (+ QFCP) and finally light quark chemical potential variation for QFCP excluded and included cases (practically coincident).

(C) 2011 IACS 